\title{
Bemerkung zu einer Arbeit von Marshall und Olkin*
}

\author{
L. EXSNER
}

Eingegangen am 10. November 1968

In [1] geben MARShaLL und OLKIN einen neuen Beweis dafür an, daB voll irreduzible (englisch: fully indecomposable, [1]) nichtnegative Rechtecksmatrizen stets so skaliert werden können, daB alle Zeilen- und Spaltensummen untereinander gleich sind. An entscheidender Stelle wird dabei das folgende Ergebnis benötigt:

Satz. Es sei $A \geqq 0$ eine voll irreduzible $m \times n$-Matrix, $\left(x^{v}, y^{v}\right)$ eine Folge mit

$$
x^{v} \in R_{m}, \quad x^{v}>0, \quad \prod_{i=1}^{m} x_{i}^{v}=1, \quad y^{v} \in R_{n}, \quad y^{v}>0, \quad \prod_{i=1}^{n} y_{i}^{v}=1
$$

Ist dann (mit $\left.\|x\|=\operatorname{Max}\left|x_{i}\right|\right) \lim \left(\left\|x^{v}\right\|+\left\|y^{v}\right\|\right)=\infty$, so gilt auch

$$
\lim _{v \rightarrow \infty} x^{v^{\prime}} A y^{v}=\infty
$$

Dieses Resultat kann einfacher und kürzer als in [1] bewiesen werden. Außerdem ist es möglich, eine untere Schranke für das Wachstum von $x^{\prime} A y$ anzugeben. Zunächst wird ein Lemma benötigt:

Lemma. Es sei $B \geqq 0$, eine voll irreduzible $m \times m$-Matrix, $b>0$ der kleinste nichtverschwindende Koeffizient von $B$. Dann gilt für alle $x>0, y>0$ mit $\prod x_{i} \geqq 1, \Pi y_{i} \geqq 1$

$$
\left(x^{\prime} B y\right)^{m+1} \geqq b^{m+1}\left(\sum_{i} x_{i}\right)\left(\sum_{i} y_{i}\right)
$$

Beweis. Da $B$ voll irreduzibel ist, kann angenommen werden, da $B$ irreduzibel ist und alle $b_{i i}>0$ sind ([1], Lemma 6). Es folgt $B^{m-1}>0$ (s. [2], S. 41). Definieren wir $H=\left(h_{r s}\right)$ durch $(x B y)^{m+1}=x^{\prime} B y x^{\prime} \ldots y x^{\prime} B y=x^{\prime} H y$, so genügt es $z u$ zeigen, daß $h_{r s} \geqq b^{m+1}$ für alle $r, s$ gilt.

$\mathrm{Zu}$ gegebenem $r$, sei $k$ mit $0 \leqq k \leqq m-1$ die kleinste Zahl mit $\left(B^{k}\right)_{r s}>0$. Wir unterscheiden zwei Fälle:

Fall $1 . k=0,1$. Es ist $b_{r s}>0$. Wir setzen

$$
W_{r s}=b_{r s} y_{s} x_{\pi_{z}} b_{\pi_{3} \pi_{s}} y_{\pi_{2}} x_{\pi_{s}} \ldots y_{\pi_{r n}} x_{s} b_{s s},
$$

wobei $\pi_{2} \ldots \pi_{m}$ eine Permutation der Zahlen $1, \ldots, s-1, s+1, \ldots, m$ ist. Da alle $x_{i}$ und $y_{i}$ genau einmal vorkommen und deren Produkt $\geqq 1$ ist, gilt $w_{r s} \geqq b^{m+1}$.

* Diese Arbeit entstand während eines Aufenthalts beim National Research Institute for Mathematical Sciences P.O. Box 395. Pretoria, South Africa. 
Fall 2. $k \geqq 2$. Es gibt $\pi_{1} \ldots \pi_{k-1}$ mit $b_{r \pi_{1}} b_{\pi_{1} \pi_{2}} \ldots b_{\pi_{k-1} s}>0$. Weil $k$ minimal ist, gilt $\pi_{i} \neq \pi_{j}$ für $i \neq j$. Mit einer Permutation $\pi_{k} \ldots \pi_{m}$ von $\{1,2, \ldots, m\} \div$ $\left\{\pi_{1}, \ldots, \pi_{k-1}\right\}$ setze

$$
w_{r s}=b_{r \pi_{1}} y_{\pi_{1}} x_{\pi_{1}} b_{\pi_{1} \pi_{z}} \ldots b_{\pi_{k-2} \pi_{k-1}} y_{\pi_{k-1}} x_{\pi_{k}} b_{\pi_{k} \pi_{k}} \ldots y_{\pi_{m}} x_{\pi_{k-1}} b_{\pi_{k-1} s}
$$

Wiederum ist $w_{r s} \geqq b^{m+1}$.

In beiden Fällen ist $w_{r s}$ ein Summand in der Darstellung

$$
h_{r s}=\sum_{i_{1} \ldots i_{2 m}} b_{r i_{1}}\left(y x^{\prime}\right)_{i_{1} i_{s}} b_{i_{z} i_{s}} \ldots\left(y x^{\prime}\right)_{i_{2 m-1}, i_{2} m} b_{i_{2} m, s} .
$$

Daher ist $h_{r s} \geqq w_{r s} \geqq b^{m+1}$. Q.e.d.

Beweis des Satzes. Es sei $m \leqq n$. Ist $y_{1} \ldots y_{n}$ mit $I I y_{i}=1, y_{i}>0$ gegeben, so fassen wir die $m$ größten $y_{i}$ zu einem Vektor $\tilde{y}$ zusammen. Es ist $\prod_{i=1}^{m} \tilde{y}_{i} \geqq 1$. $B$ sei die voll irreduzible Matrix, die durch Streichen aller Spalten, deren Index in $y$, aber nicht in $\tilde{y}$ vorkommt, aus $A$ entsteht. Ist $a$ der kleinste nichtverschwindende Koeffizient von $A$, so gilt $b \geqq a$. Wir wenden das Lemma an und erhalten

$$
\left(x^{\prime} A y\right)^{m+1} \geqq\left(x^{\prime} B \tilde{y}\right)^{m+1} \geqq a^{m+1}\left(\sum x_{i}\right)\left(\sum \tilde{y}_{i}\right) \text {. }
$$

Wegen $\|y\|=\|\tilde{y}\|$ geht auch $\left\|x^{v}\right\|+\left\|\tilde{y}^{v}\right\|$ gegen Unendlich. Aus (1) folgt nun die Behauptung sofort.

Bemerkung 1. Offenbar gilt

$$
x^{\prime} A y \geqq a \cdot\|x\|^{\frac{1}{m+1}}\|y\|^{\frac{1}{m+1}} .
$$

Bemerkung 2. Die aus (2) folgende globale untere Schranke $x^{\prime} A y \geqq a$ kann leicht verbessert werden:

$$
x^{\prime} A y \geqq x^{\prime} B \tilde{y} \geqq \min \left(b_{i i}\right) \cdot \sum x_{j} \tilde{y}_{j} \geqq a \cdot m \cdot \prod x_{i}^{1 / m} \prod \tilde{y}_{i}^{1 / m} \geqq m \cdot a .
$$

\section{Literatur}

1. Marshall, A. W., and T. Olkin: Scaling of matrices to achieve specified row and column sums. Num. Math. 12, 83-90 (1968).

2. VARGA, R. S.: Matrix iterative analysis. London: Prentice-Hall 1962.

L. ELSNER

Institut für angewandte Mathematik der Universität

2000 Hamburg 13

Rothenbaumchaussee 67/69 